\title{
Evaluation of cytokines in peripheral blood mononuclear cell supernatants for the diagnosis of tuberculosis
}

This article was published in the following Dove Press journal: Journal of Inflammation Research

\author{
Margaretha Sariko ${ }^{1-3}$ \\ Athanasia Maro ${ }^{1,3}$ \\ Jean Gratz ${ }^{1,4}$ \\ Eric Houpt ${ }^{4}$ \\ Riziki Kisonga ${ }^{1,5}$ \\ Stellah Mpagama ${ }^{1,5}$ \\ Scott Heysell ${ }^{4}$ \\ Blandina T Mmbaga ${ }^{1-3, *}$ \\ Tania A Thomas ${ }^{4, *}$ \\ 'Kilimanjaro Clinical Research \\ Institute, Moshi, Tanzania; ${ }^{2}$ Kilimanjaro \\ Christian Medical University College, \\ Moshi, Tanzania; ${ }^{3}$ Kilimanjaro Christian \\ Medical Centre, Moshi, Tanzania; \\ ${ }^{4}$ Division of Infectious Diseases and \\ International Health, Department \\ of Medicine, University of Virginia, \\ Charlottesville, VA, USA; ${ }^{5}$ Kibong'oto \\ Infectious Diseases Hospital, \\ Kilimanjaro, Tanzania \\ *These authors contributed equally to \\ this work
}

Correspondence: Margaretha Sariko Kilimanjaro Clinical Research Institute, P.O. Box 2236, Moshi, Tanzania

Tel +25527275 4201

Fax +255272753368

Email m.sariko@kcri.ac.tz
Introduction: There is active interest in leveraging host immune responses as biomarkers of tuberculosis (TB) disease activity. We had previously evaluated an immunodiagnostic test called the antibody in lymphocyte supernatant (ALS) assay. Here, we aimed to evaluate a panel of inflammatory mediators and associate the responses with the ALS results to identify a biosignature to distinguish TB cases from controls.

Methodology: In this case-control study, adults with TB were compared to controls who were hospitalized for non-infectious conditions. Blood was collected at baseline and after 4 weeks of TB treatment (from TB cases only). Peripheral blood mononuclear cells were isolated and cultured without antigenic stimulation for 72 hours. Inflammatory mediators were measured using the Multiplex cytokine kit and compared between TB cases and controls; among TB cases, responses were compared over time. ALS and inflammatory mediator results were evaluated using generalized discriminant analysis to identify the optimal biosignature to predict TB.

Results: When comparing inflammatory mediators between groups, IL-1ra, IL-1 $\beta$, and granulocyte macrophage-colony stimulating factor (GM-CSF) were lower in TB cases $(P<0.002)$. Fibroblast growth factor-basic significantly increased from baseline to week-4 $(P=0.002)$. Generalized discriminant analysis yielded a model with IL-2, tumor necrosis factor-alpha, vascular endothelial growth factor, and ALS, providing a sensitivity of $82.2 \%$ and specificity of $76.2 \%$.

Conclusion: Our results suggest that IL-1ra, IL-1 $\beta$, and GM-CSF might be used as diagnostic biomarkers to distinguish between TB cases and non-TB cases. We could not identify a group of mediators that outperformed the diagnostic accuracy of the ALS alone.

Keywords: cytokines, chemokines, biomarkers, TB, diagnostics

\section{Introduction}

Tuberculosis (TB) remains a global threat to human health. It is the leading cause of death from a single bacterial pathogen, contributing to the demise of 1.7 million persons in 2016. ${ }^{1}$ TB diagnosis is still a challenge, and improved diagnostic methods are a key part of achieving the End TB Strategy goals. ${ }^{2}$ There is active interest in leveraging host immune responses as biomarkers of disease activity which builds upon the growing understanding of the mutually supportive roles between innate and adaptive immune responses in the control of TB.,

Plasmablasts are immature antibody-secreting cells, which have been used as the basis for a novel blood-based TB diagnostic biomarker, the antibody in lymphocyte supernatant (ALS) assay. ${ }^{5}$ ALS measures the incident secretion of mycobacterial antibodies from plasmablasts within peripheral blood mononuclear cells (PBMCs) that 
are isolated and cultured without antigenic stimulation. The ALS assay has been studied as a TB diagnostic among adults from Bangladesh and Ethiopia, including HIV co-infected adults. $^{5-11}$ We examined the performance of this assay in Tanzania and found that it was $92 \%$ sensitive and $96 \%$ specific in predicting adults with TB vs hospitalized controls. ${ }^{12}$ Additionally, B-cells can act as antigen-presenting cells that trigger further proliferation and differentiation of T-cells as well as the generation of plasmablasts. ${ }^{3,6}$

Cytokines and chemokines are inflammatory mediators that are produced throughout the body by cells of diverse embryological origin. ${ }^{13}$ Upon encountering Mycobacterium tuberculosis (MTB) in the pulmonary alveoli, innate immune responses are triggered when antigen-presenting cells produce inflammatory mediators that work to coordinate early host immune defense mechanisms. These inflammatory mediators include pro-inflammatory cytokines such as tumor necrosis factor-alpha (TNF- $\alpha$ ) and interferongamma (IFN- $\gamma$ ), cytokines such as IL-12 and IL-23 which through the priming and polarization of T-helper cells orchestrate important roles for granuloma assembly and anti-inflammatory cytokines such as IL-10 and transforming growth factor beta which have the ability to inhibit the synthesis of the major inflammatory mediators. Chemokines include CXCL-3 macrophage inflammatory protein ([MIP]-2 $\beta$ ) and CXCL-5 (ENA-78) which are associated with recruitment of neutrophils and natural killer cells. ${ }^{14-17}$ Understanding the inflammatory mediators in the early course of TB may provide insight into the development of immunologic biomarkers of disease activity.

Here, we present an evaluation of 24 cytokines, chemokines, and growth factors detected from unstimulated PBMC supernatants, with the aim of building upon the diagnostic accuracy of the ALS assay among our previously described cohort of adults from Tanzania with TB and TB/HIV. ${ }^{12}$ Also, we evaluate if these inflammatory mediators can be used as an early treatment-monitoring tool by comparing the difference between levels measured at baseline and 4 weeks after the initiation of TB treatment.

\section{Materials and methods Study design and population}

This is a sub-study of our recent investigation of the ALS assay. ${ }^{12}$ Briefly, this was a case-control study conducted in the Kilimanjaro region of Tanzania in which adults initiating treatment for pulmonary TB were compared to hospitalized controls without TB.
Adults presenting symptoms of pulmonary TB were recruited from Kibong'oto Infectious Disease Hospital, the national referral hospital for multi-drug resistant TB (MDR-TB) in Kilimanjaro, Tanzania. Eligibility criteria were as previously described. ${ }^{12}$ Briefly, "confirmed TB cases" included people with MTB detected from sputum by GeneXpert MTB/RIF (Cepheid, Sunnyvale, CA, USA) ${ }^{18}$ or by TB culture; "clinically diagnosed cases" included those who did not have a microbiological diagnosis, but were initiated on TB treatment based on clinical grounds and responded to therapy. All cases were treated with first- or second-line anti-TB drugs according to their drug-sensitivity profiles and national guidelines by the physician treating them. ${ }^{19}$ Blood samples were collected at baseline and at 4 weeks after the initiation of TB therapy.

Adults who were hospitalized due to non-infectious conditions such as diabetes, hypertension, abdominal pain, and gastrointestinal bleeding were recruited as controls from Kilimanjaro Christian Medical Center Hospital, as previously described. ${ }^{12}$ Briefly, we verbally screened control participants to ensure that they had no current or prior treatment for TB, no signs or symptoms consistent with TB, and no prior contact with a known TB case; attempts were made to age-match controls with cases ( \pm 5 years). Cases and controls were included in this sub-study if they had sufficient PBMC supernatants available for analysis.

\section{Sample processing}

Overnight-pooled sputum samples were collected from the TB cases and transported to Kilimanjaro Clinical Research Institute. ${ }^{20}$ The collected sputum sample was processed for GeneXpert MTB/RIF and culture, both liquid and solid TB cultures. $^{18,21}$

\section{PBMC culture and inflammatory mediator measurements}

As previously described, the ALS assay was conducted from the supernatants of PBMCs that were isolated and cultured without antigenic stimulation or any other cell receptor blockers for 72 hours to allow for maximum expression of all mediators. ${ }^{5,12,22}$ For protection against contamination, tissue cultures included the use of heat-inactivated bovine serum and L-glutamine; penicillin and streptomycin were also added to the culture media. Residual supernatants from the PBMC culture were frozen at $-80^{\circ} \mathrm{C}$ with the protease inhibitor for batch analysis. The ALS assay was an ELISA based assay that used freezedried glutamate bacille Calmette-Guérin vaccine (Japan BCG 
Laboratories, Tokyo, Japan) as the coating antigen and captured IgG antibodies from the supernatant.

Cytokines, chemokines, and growth factors from the supernatants were measured in duplicate using the Bio-Plex Pro Human Cytokine Multi-Plex, Group I kit (Bio-Rad Laboratories Inc., Hercules, CA, USA) according to the manufacturer's instructions on the Luminex platform (Bio-Plex 200, Bio-Rad Laboratories Inc.). ${ }^{23}$ Briefly, the plate was pre-wet using $100 \mu \mathrm{L}$ of Bio-Plex assay buffer, and $50 \mu \mathrm{L}$ of diluted magnetic beads coupled with capture antibody were added. Then, $50 \mu \mathrm{L}$ of undiluted supernatant was added. Then, $50 \mu \mathrm{L}$ of the supernatant was added without dilution. The secondary antibody was added after removal of unbound antigens and lastly, the conjugate was added. The beads were re-suspended using $125 \mu \mathrm{L}$ of assay buffer. Analyte values were calculated based on standard curves as per the manufacturer's instructions. Three mediators were excluded from the analysis because the majorities were out of range and re-analysis with dilutions could not be performed. The following inflammatory mediators met quality control procedures and were included in the analysis: IL-1 $\beta$, IL-1 receptor antagonist (IL-1ra), IL-2, IL-4, IL-5, IL-7, IL-8, IL-9, IL-10, IL12p70, IL-13, IL-17A, eotaxin, fibroblast growth factor-basic (FGF-basic), granulocyte-colony stimulating factor (G-CSF), granulocyte macrophage-colony stimulating factor (GM-CSF), IFN- $\gamma$, inducible protein-10 (IP-10), monocyte chemoattractant protein-1 (MCP-1), macrophage inflammatory protein-1 $\alpha$ (MIP-1 $\alpha$ ), monocyte inducible protein-1 $\beta$ (MIP-1 $\beta$ ), platelet derived growth factor-bb (PDGF-bb), TNF- $\alpha$, and vascular endothelial growth factor (VEGF).

\section{Ethical approval}

Ethical clearance was obtained from the National Institute for Medical Research, Kilimanjaro Christian Medical University College ethical committee, and University of Virginia. All participants provided written informed consent. The study was conducted in accordance with the principles set forth in the Declaration of Helsinki.

\section{Data analysis}

Descriptive analyses, simple frequencies, and the distribution of results were evaluated. Non-parametric independent Mann-Whitney $U$-tests were used to compare the levels of inflammatory mediators between groups. Among TB cases, longitudinal responses from baseline and week 4 of treatment were compared using the Wilcoxon-signed rank test. General discriminant analysis was used to build a model of inflammatory mediators including ALS results to improve the predictive ability for TB. The data were analyzed using Statistical Package for Social Science (Version 22.0, International Business
Machines, Inc, Chicago, IL, USA), and graphs were drawn using GraphPad Prism6 (GraphPad Software, La Jolla, CA, USA). The Bonferroni correction was applied to a $P$-value of 0.05 to account for multiple testing; a $P$-value of 0.002 was considered significant in our analyses.

\section{Results \\ Demographic characteristics}

Between January 2014 and May 2015, 125 participants were enrolled in the parent study; 94 (75\%) had sufficient supernatant for analysis in this sub-study, including 73 TB cases and 21 hospitalized controls. Among the TB cases, 44 (60\%) had drug-susceptible TB, 22 (30\%) had MDR-TB, and $7(10 \%)$ were clinically diagnosed. HIV co-infection was prevalent in $20(27 \%)$ TB cases, $14(70 \%)$ of whom were on anti-retroviral treatment. Among all study participants, four were known to have diabetes and were receiving diabetes treatment, three $(75 \%)$ of whom were hospitalized controls Additional demographic details are displayed in Table 1.

\section{Inflammatory mediators from unstimulated PBMCs among TB cases and controls}

Participants with TB had significantly lower levels of IL-1ra $(P=0.001), \mathrm{IL}-1 \beta(P=0.002)$ (Figure 1B), and GM-

Table I Demographic characteristics of the study participants

\begin{tabular}{|l|l|l|}
\hline Characteristics & $\begin{array}{l}\text { Controls } \\
\mathbf{n = 2 I}(\%)\end{array}$ & $\begin{array}{l}\text { TB cases } \\
\mathbf{n = 7 3}(\%)\end{array}$ \\
\hline $\begin{array}{l}\text { Age, mean in years } \pm \text { SD } \\
\text { Gender }\end{array}$ & $4 I \pm I 3.5$ & $39.8 \pm 12.7$ \\
Male & $4(19)$ & $59(81)$ \\
Female & $17(8 I)$ & $14(19)$ \\
HIV status & & \\
Positive & $3(14)$ & $20(27)$ \\
Negative & $13(62)$ & $53(73)$ \\
Unknown & $5(24)$ & \\
BMI & & \\
Normal, BMI $\geq 18.5$ & $16(76)$ & $44(60)$ \\
Malnourished, $<18.5$ & 0 & $29(40)$ \\
Unknown & $5(24)$ & \\
Method of TB diagnosis & N/A & \\
GeneXpert & & $32(44)$ \\
Culture and DST & & $34(46)$ \\
Clinically diagnosed & & $7(10)$ \\
Final Treatment outcome & N/A & $21(29)$ \\
Cure & & $23(32)$ \\
Treatment complete & & $1(1)$ \\
Failed & & $11(15)$ \\
Died & & $14(19)$ \\
Defaulted & & \\
Transfer out/unknown & & \\
\hline
\end{tabular}

Abbreviations: BMI, body mass index; DST, drug susceptible testing; NA, not applicable. 

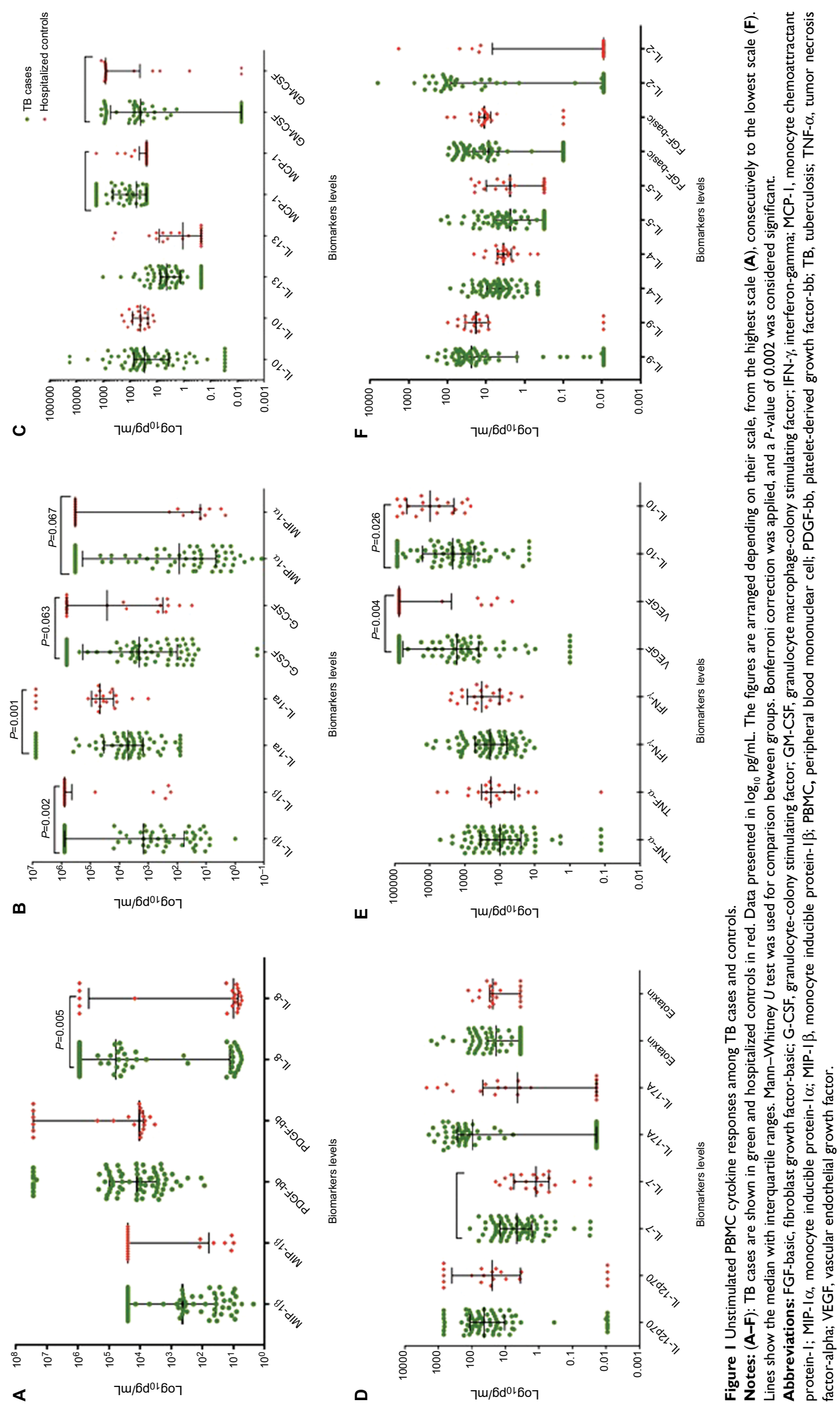

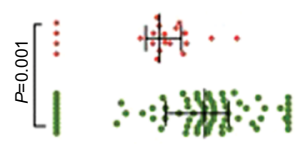

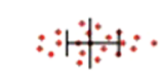

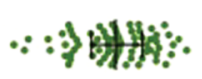

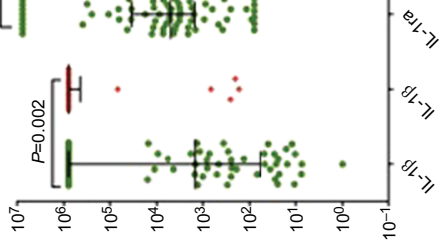

m

ךแ/6d 01607

ш

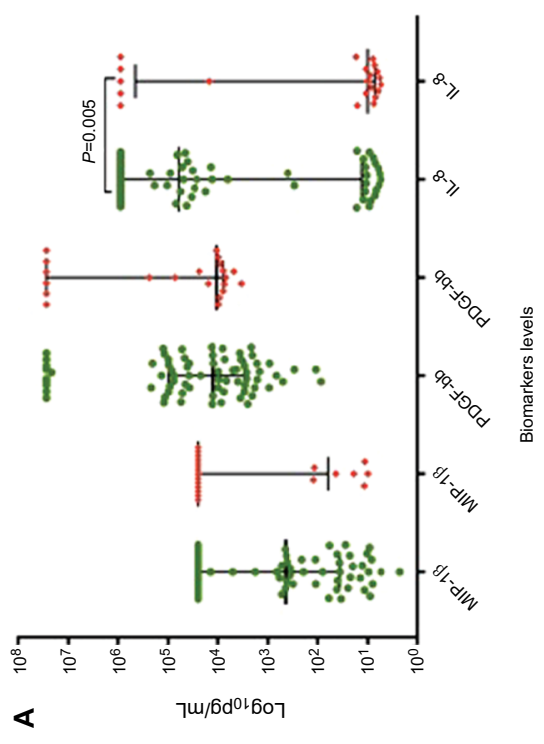

w

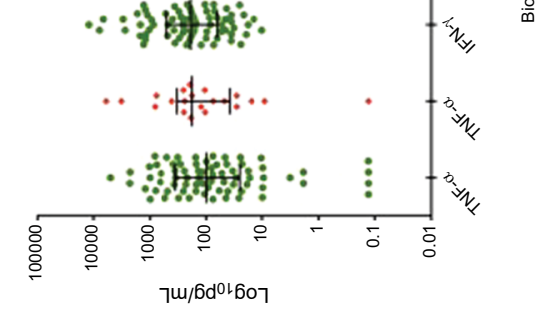

口

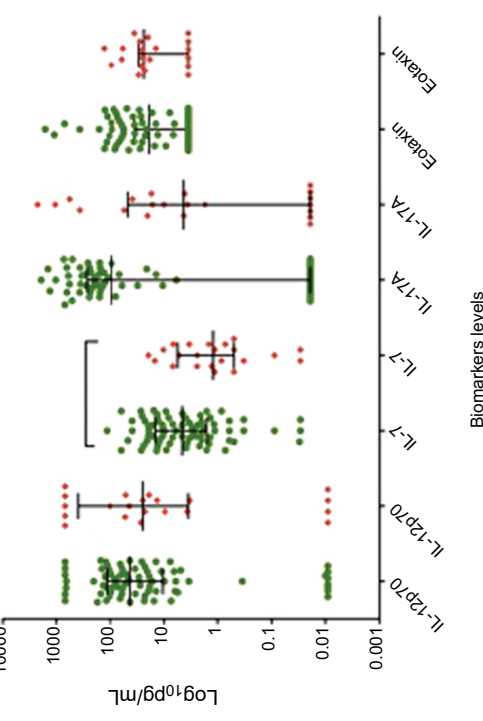

my 
CSF $(P=0.002)$ (Figure 1C). MIP-1 $\beta(P=0.119)$, G-CSF $(P=0.063)$, MIP-1 $\alpha(P=0.067)$, and IP-10 $(P=0.026)$ were low when compared to hospitalized controls but not significant. Most mediators did not show a significant difference. Median values among TB cases did not significantly differ by HIV status (data not shown).

\section{Inflammatory mediator responses among TB cases before and after 4 weeks of treatment}

In the longitudinal comparison of responses of inflammatory mediators among participants with TB, most did not show any significant change over time. The only inflammatory mediator that significantly changed over time was fibroblast growth factor-basic (FGF-basic), which showed an increase after 4 weeks of TB treatment when compared to baseline levels $(P=0.002)$ (Figure 2).

\section{Association between ALS levels and inflammatory mediator levels in the identification of TB cases}

General discriminant analysis showed that IL-2, TNF $\alpha$, and VEGF with ALS offered the optimal combined profile as a diagnostic ( $83 \%$ sensitivity, $81 \%$ specificity); however, this profile was no better than ALS alone (92\% sensitivity, 96\% specificity). ${ }^{12}$

\section{Discussion}

The host immune response to bacterial invasion is key to determine the status of the infection, if it is controlled
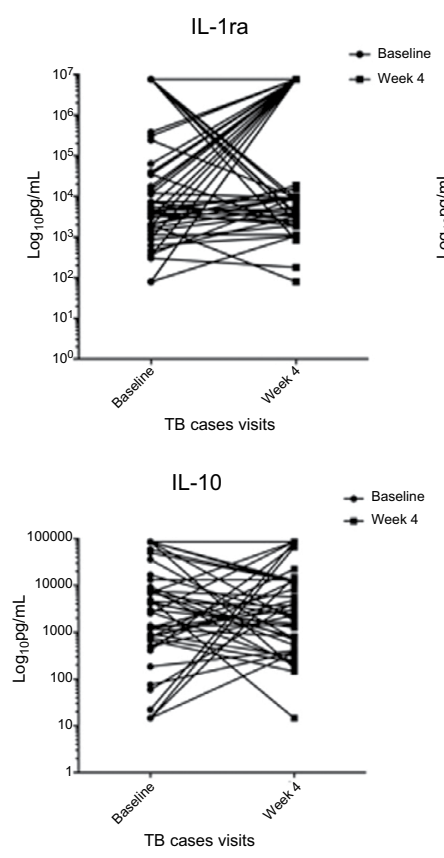

IL-7

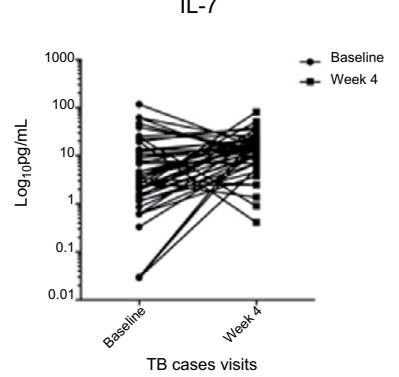

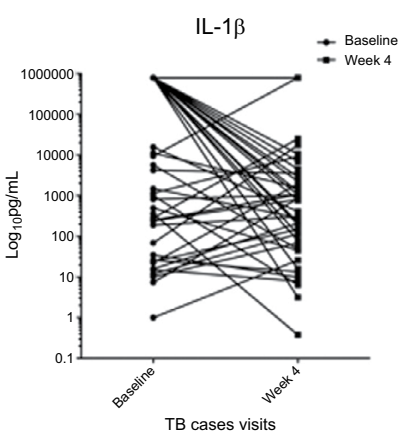

TB cases visits

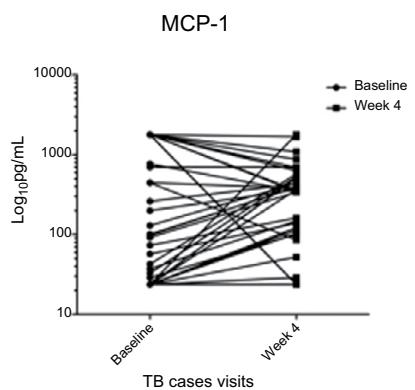

TB cases visits

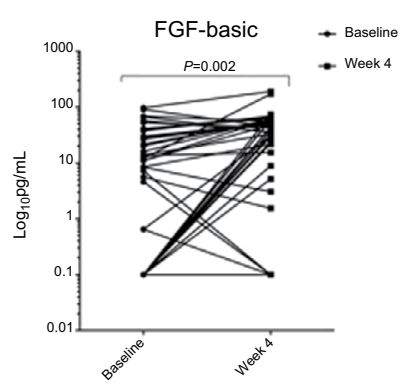

VEGF

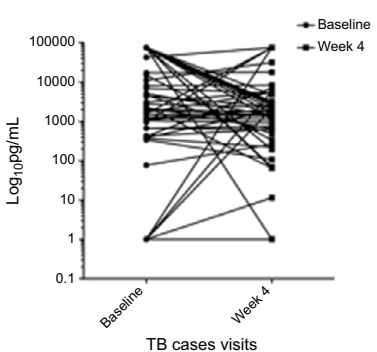

IL-13

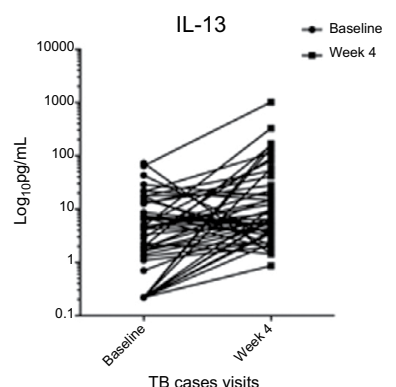

TB cases visits

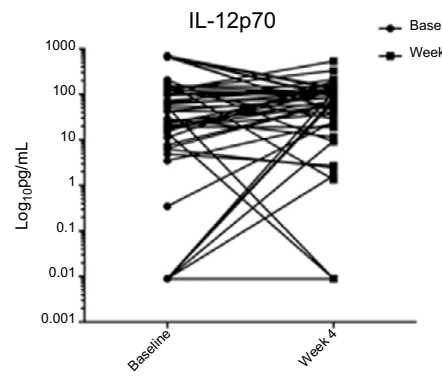

TB cases visits
IFN- $\gamma$
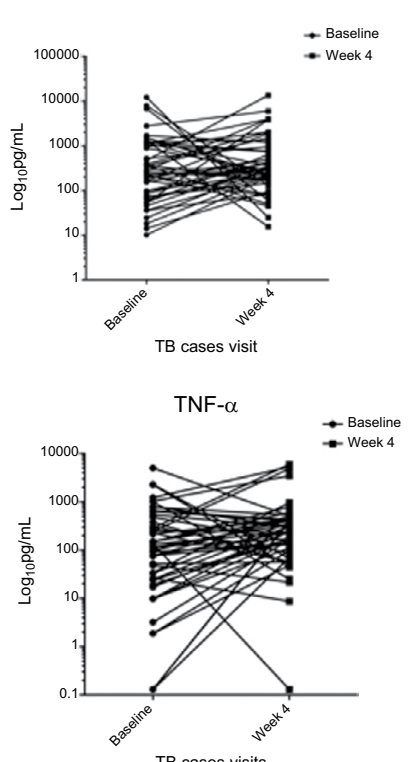

TB cases visits

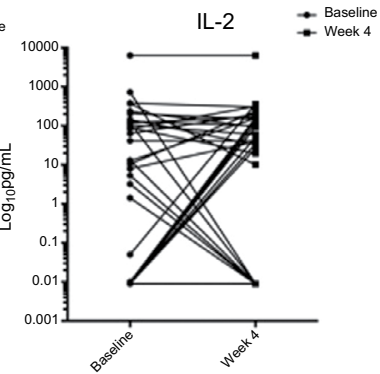

TB cases visits

Figure 2 Comparison of cytokine levels among TB cases before and after 4 weeks of TB treatment.

Notes: Figures depicting the longitudinal changes of cytokine, chemokine, and growth factor expression among TB cases, $\mathrm{n}=73$. Concentrations are shown at baseline and at week 4 of treatment and results are expressed as $\log _{10} \mathrm{Pg} / \mathrm{mL}$. Statistical analyses were performed using non-parametric Wilcoxon paired test and a $P=0.002 \mathrm{was}$ considered significant. FGF-basic significantly increased over time $(P=0.002)$.

Abbreviations: TNF- $\alpha$, tumor necrosis factor-alpha; FGF-basic, fibroblast growth factor-basic; MCP-I, monocyte chemoattractant protein-I; TB, tuberculosis; TNF- $\alpha$, tumor necrosis factor-alpha; VEGF, vascular endothelial growth factor. 
Table 2 Association of ALS and other cytokines in predicting TB cases and hospitalized controls

\begin{tabular}{|l|l|l|l|l|l|l|}
\hline Biomarkers & $\begin{array}{l}\text { Number of } \\
\text { participants }\end{array}$ & Case status & Sensitivity (\%) & Specificity (\%) & PPV (\%) & NPV (\%) \\
\hline $\begin{array}{l}\text { ALS, VEGF, TNF- } \alpha, \\
\text { and IL-2 }\end{array}$ & 66 & $\begin{array}{l}\text { Confirmed TB } \\
\text { cases }\end{array}$ & 82.8 & 81.0 & 93.0 & 60.7 \\
\hline $\begin{array}{l}\text { ALS, VEGF, TNF- } \alpha, \\
\text { and IL-2 }\end{array}$ & 73 & All TB cases & 82.2 & 76.2 & 92.3 & 55.2 \\
\hline
\end{tabular}

Note: All TB cases - this includes microbiologically confirmed cases $(n=66)$ and clinically diagnosed cases $(n=7)$.

Abbreviations: NPV, negative predictive values; PPV, positive predictive values; ALS, antibody in lymphocyte supernatant; TNF- $\alpha$, tumor necrosis factor-alpha; VEGF, vascular endothelial growth factor; TB, tuberculosis.

or not. Innate immune response and adaptive immune response play a key role in MTB control. ${ }^{24}$ The outcome of the infection is dependent on the protective power of the host's immune system and the pathogenicity of the bacteria. Innate immunity is by phagocytosis and subsequent release of IL-12 in the absence of memory exposure to the antigen of interest. The other component of innate immunity is the natural resistance associated with macrophage proteins like natural killer cells and neutrophils. Adaptive immunity is triggered when the bacterial infection eludes the innate defense mechanisms that may be humoral by antibody production and cell-mediated immunity through effector $\mathrm{T}$ cells. ${ }^{25,26} \mathrm{~T}$ cells are categorized in three groups: Th1 which secrete IL- 2 and IFN- $\gamma$ and play a role in intracellular infections, Th2 which secrete IL-4, IL5, and IL-10 which enhance antibody synthesis of B cells ${ }^{27}$ and Th17 which release IL-17 manipulated by MTB microorganisms for their survival benefits in primary TB. ${ }^{28}$

In this work, we compare the inflammatory mediator responses between TB patients and hospitalized controls using supernatants from PBMC cultures. Measuring secreted cellular byproducts from culture supernatants has shown potential, as evidenced by our work using ALS assays for the diagnosis of TB. We found that TB cases had significantly lower levels of the following inflammatory cytokines: IL1ra, IL-1 $\beta$, and GM-CSF. However, none of the measured mediators could improve upon the diagnostic accuracy of the ALS assay.

IL-1ra is an anti-inflammatory cytokine produced by monocytes, macrophages, and dendritic cells. ${ }^{29}$ During infections, including TB, levels of anti-inflammatory cytokines are often found to be depressed. Indeed, in our cohort, we detected lower IL-1ra levels among those in the TB group compared to hospitalized controls. When we re-evaluated the IL-1 ra levels after 4 weeks of TB treatment, we had expected a rise in IL-1ra levels; however, a significant change was not detected $(P=0.32)$. Other studies that have examined the changes in serum IL-1ra over multiple time points after TB treatment initiation have also found non-significant fluctuations throughout the treatment course..$^{29}$

Two pro-inflammatory cytokines were noted to be significantly different between our groups: IL- $1 \beta$ and GMCSF. Because the untreated stage of TB typically prompts an avid inflammatory response, we had hypothesized that the pro-inflammatory cytokine should be found at higher concentrations in TB cases compared to the control population. However, we detected lower IL-1 $\beta$ levels among the TB group. IL-1 $\beta$ is produced by monocytes, macrophages, and dendritic cells and is down regulated by IL-1ra. Given the known antagonistic roles of IL- $1 \beta$ and IL-1 $1 \mathrm{ra},{ }^{30,31}$ it is possible that the comparatively higher levels of IL-1ra seen within the TB group compared to IL- $1 \beta$ in the same TB group were influenced by the antagonistic relationship between IL-1 $\beta$ and IL-1ra.

GM-CSF is a growth factor that serves as a chemoattractant by recruiting neutrophils, lymphocytes, and macrophages. GM-CSF is involved in supporting the productive phase of inflammatory responses. ${ }^{22,32} \mathrm{In} \mathrm{TB}$, it plays role in granuloma formation to contain the organism. ${ }^{33}$ In our cohort, TB cases had lower GM-CSF levels compared to hospitalized controls. We are aware that TB has the ability to affect the expression of GM-CSF though the mechanism is not known. ${ }^{34}$

Interestingly, we did not detect any differences among the mediators that have been studied most as TB biomarkers, such as IFN- $\gamma$, TNF- $\alpha$, and IP-10. IFN- $\gamma$ is one of the important mediators in cell-mediated immune responses; it is produced by activated T-cells, ${ }^{35}$ plays a major role in activating macrophages, and assists in killing intracellular organisms. TNF- $\alpha$ is a mediator produced by monocytes and macrophages that assists in killing intracellular organisms by signaling apoptosis. ${ }^{36,37} \mathrm{IP}-10$ is a chemokine that is produced by antigen-presenting cells. Traditionally, studies reporting elevated levels of IFN- $\gamma$, TNF- $\alpha$, and IP-10 in TB cases have measured responses from plasma or supernatant 
after stimulation with TB-specific antigens. ${ }^{35,38,39}$ It is possible that our findings differ from what has been previously reported in literature because we have used PBMC culture supernatants that have not been stimulated by TB-specific antigens. Similarly, we did not detect any difference in mediator levels between participants with and without HIV co-infection. The majority (70\%) of people co-infected with HIV were on antiretroviral therapy, which may have affected our ability to detect a difference in mediator responses between these sub-groups. When comparing mediator levels after initiation of TB treatment, most mediators did not show a significant difference aside from FGF-basic. FGF-basic is produced by macrophages and plays a role in mitogenic and angiogenic activity. ${ }^{40}$ With respect to TB, FGF-basic has not demonstrated a consistent association with the disease when examining its stimulated or unstimulated levels. ${ }^{41,42}$ Another study, examining the trends in FGF-basic levels over time among adults with TB from Africa, did not reveal any consistent changes. ${ }^{43}$

In our attempt to combine mediator responses with ALS results, we found that ALS was the strongest predictor for TB status. When used alone, ALS could categorize TB cases and hospitalized controls with a sensitivity of $92 \%$ and specificity of $96 \%,{ }^{12}$ our optimal combination of mediators and ALS did not outperform this.

Our study had limitations including the relatively short follow-up interval among TB cases (4 weeks) compared to the total duration of TB treatment. This may have hindered our ability to detect a longitudinal difference in mediator levels, especially among participants who had MDR-TB and may have experienced a slower immunologic response. Extended follow-up intervals could provide more insight into the trends of mediator levels. Some of our mediator responses exceeded the quantifiable range, which may have affected our ability to detect a true difference in levels between the groups. Serial dilution of the samples to obtain the actual reading could have mitigated this; however, this was not possible due to limited specimen volumes. Specimens used to conduct this study were also used for our first study examining the performance of the ALS assay. It is important to note that samples were not subjected to multiple freeze-thaw cycles and that storage conditions were the same for all specimens; therefore, we do not believe that mediator degradation could have differentially affected our results.

\section{Conclusion}

In sum, few studies test unstimulated supernatant for mediator biomarkers to improve the diagnosis of TB. We found that the supernatant levels of IL-1ra, IL-1 $\beta$, and GM-CSF could potentially be used to discriminate $\mathrm{TB}$ cases from hospitalized controls. These results may contribute to new mediator-based diagnostics for TB.

\section{Acknowledgments}

We are grateful to the study participants and research staff who helped to make these important findings possible. NIH D43 TW008270 and D43 TW006578 NIH/Fogarty International Center Global Infectious Disease Research Training Program at UVA supported this work. MS is supported by COSTECH - Commission of Science and Technology, Tanzania and GIDRT fund through Centre of Global Health in UVA. NIH/NIAID K23-AI097197 supports TAT.

\section{Author contributions}

All authors contributed to data analysis, drafting or revising the article, gave final approval of the version to be published, and agree to be accountable for all aspects of the work.

\section{Disclosure}

The authors report no conflicts of interest in this work.

\section{References}

1. Global Tuberculosis Report. Tuberculosis. 2017. Available from: http:// apps.who.int/iris/bitstream/10665/259366/1/9789241565516-eng. pdf?ua $=1$. Accessed November 27, 2017.

2. World Health Organization. EndTB Strategy. 2015. Available from: https:// www.who.int/tb/End_TB_brochure.pdf. Accessed November 27, 2017.

3. Kozakiewicz L, Phuah J, Flynn J, Chan J. The role of B cells and humoral immunity in Mycobacterium tuberculosis infection. Adv Exp Med Biol. 2013;783:225-250.

4. Wallis RS, Pai M, Menzies D, et al. Biomarkers and diagnostics for tuberculosis: progress, needs, and translation into practice. Lancet. 2010;375(9729):1920-1937.

5. Raqib R, Rahman J, Kamaluddin AK, et al. Rapid diagnosis of active tuberculosis by detecting antibodies from lymphocyte secretions. $J$ Infect Dis. 2003;188(3):364-370.

6. Shen P, Fillatreau S. Antibody-independent functions of B cells: a focus on cytokines. Nat Rev Immunol. 2015;15(7):441-451.

7. Ashenafi S, Aderaye G, Zewdie M, et al. BCG-specific IgG-secreting peripheral plasmablasts as a potential biomarker of active tuberculosis in HIV negative and HIV positive patients. Thorax. 2013;68(3):269-276.

8. Rekha RS, Kamal SM, Andersen P, et al. Validation of the ALS assay in adult patients with culture confirmed pulmonary tuberculosis. PLoS One. 2011;6(1):e16425.

9. Raqib R, Kamal SM, Rahman MJ, et al. Use of antibodies in lymphocyte secretions for detection of subclinical tuberculosis infection in asymptomatic contacts. Clin Diagn Lab Immunol. 2004;11(6):1022-1027.

10. Raqib R, Mondal D, Karim MA, et al. Detection of antibodies secreted from circulating Mycobacterium tuberculosis-specific plasma cells in the diagnosis of pediatric tuberculosis. Clin Vaccine Immunol. 2009;16(4):521-527.

11. Thomas T, Brighenti S, Andersson J, Sack D, Raqib R. A new potential biomarker for childhood tuberculosis. Thorax. 2011;66(8):727-729.

12. Sariko M, Anderson C, Mujaga BS, et al. Evaluation of the antibody in lymphocyte supernatant assay to detect active tuberculosis. PLoS One. 2017;12(1):e0169118-13. 
13. Scapigliati G, Buonocore F, Mazzini M. Biological activity of cytokines: an evolutionary perspective. Curr Pharm Des. 2014;2006(12):3071-3081.

14. Oppmann B, Lesley R, Blom B, et al. Novel p19 protein engages IL$12 \mathrm{p} 40$ to form a cytokine, IL-23, with biological activities similar as well as distinct from IL-12. Immunity. 2000;13(5):715-725.

15. Seder RA, Gazzinelli R, Sher A, Paul WE. Interleukin 12 acts directly on CD4+ $\mathrm{T}$ cells to enhance priming for interferon gamma production and diminishes interleukin 4 inhibition of such priming. Proc Natl Acad Sci U S A. 1993;90(21):10188-10192.

16. Cooper AM. Cell-mediated immune responses in tuberculosis. Annu Rev Immunol. 2009;27(1):393-422.

17. Kang DD, Lin Y, Moreno JR, Randall TD, Khader SA. Profiling early lung immune responses in the mouse model of tuberculosis. PLoS One. 2011;6(1):e16161.

18. Cepheid®. Xpert® MTB/RIF Package Insert Ref GXMTB/RIF-US-10. 2015. Available from: http://www.cepheid.com/us/cepheid-solutions/ clinical-ivd-tests/critical-infectious-diseases/xpert-mtb-rif. Accessed October 12, 2018.

19. National Tuberculosis and Leprosy Programme. Manual for the Management of TB and Leprosy 2013-2017; 2012. Available from: https://moh. gov.gm/sites/default/files Final_TB_Manual_2013-2017\%5B1\%5D_0. pdf/. Accessed November 28, 2017.

20. Mpagama SG, Mtabho C, Mwaigwisya S, et al. Comparison of overnight pooled and standard sputum collection method for patients with suspected pulmonary tuberculosis in northern Tanzania. Tuberc Res Treat. 2012;2012(128057):1-5.

21. Palomino JC, Martin A, Portaels F. Evaluation of automated BACTEC MGIT 960 system for testing susceptibility of TB. J Clin Microbiol. 2007;37(3):607-610.

22. Cheknev SB, Apresova MA, Moryakova NA, et al. Production of the growth factors GM-CSF, G-CSF, and VEGF by human peripheral blood cells induced with metal complexes of human serum $\gamma$-globulin formed with copper or zinc ions. Mediators Inflamm. 2014;2014:518265-8.

23. Instruction Manual. Bio-Plex Pro TM Human Cytokine, Chemokine, and Growth Factor Assays. Hercules, CA: Bio-Rad Laboratories, Inc.; 2009:1-47.

24. Romero-Adrian TB, Leal-Montiel J, Valecillo A. Role of cytokines and other factors involved in the Mycobacterium tuberculosis infection. World J Immunol. 2015;5(1):16.

25. Maglione PJ, Xu J, Chan J. B cells moderate inflammatory progression and enhance bacterial containment upon pulmonary challenge with Mycobacterium tuberculosis. J Immunol. 2007;178(11):7222-7234.

26. Wan YY, Flavell RA. How diverse--CD4 effector T cells and their functions. J Mol Cell Biol. 2009;1(1):20-36.

27. Surcel H-M, Troye-Blomberg M, Pauliet S, et al. Thl/Th2 profiles in tuberculosis, based on the proliferation and cytokine response of blood lymphocytes to mycobacterial antigens. Immunology. 1994;81(2);171-176.

28. Shen H, Chen ZW. The crucial roles of Th17-related cytokines/ signal pathways in M. tuberculosis infection. Cell Mol Immunol. 2018;15(3):216-225.
29. Lee JH, Chang JH. Changes of plasma interleukin-1 receptor antagonist, interleukin-8 and other serologic markers during chemotherapy in patients with active pulmonary tuberculosis. Korean J Intern Med. 2003;18(3):138-145.

30. Sasindran SJ, Torrelles JB. Mycobacterium tuberculosis infection and inflammation: what is beneficial for the host and for the bacterium? Front Microbiol. 2011;2:1-16.

31. Dinarello CA. Biologic Basis for Interleukin-1 in Disease. Blood. 1996;87(6):2095-2147.

32. Olsson AK, Dimberg A, Kreuger J, Claesson-Welsh L. VEGF receptor signalling - in control of vascular function. Nat Rev Mol Cell Biol. 2006;7(5):359-371.

33. Szeliga J, Daniel DS, Yang CH, Sever-Chroneos Z, Jagannath C, Chroneos ZC. Granulocyte-macrophage colony stimulating factor-mediated innate responses in tuberculosis. Tuberculosis (Edinb). 2008;88(1): $7-20$.

34. Cho JE, Park S, Lee H, Cho SN, Kim YS. Mycobacterium tuberculosisinduced expression of granulocyte-macrophage colony stimulating factor is mediated by PI3-K/MEK1/p38 MAPK signaling pathway. BMB Rep. 2013;46(4):213-218.

35. Xiong W, Dong H, Wang J, et al. Analysis of plasma cytokine and chemokine profiles in patients with and without tuberculosis by liquid array-based multiplexed immunoassays. PLoS One. 2016;11(2):e0148885.

36. Ehlers S. Role of tumour necrosis factor (TNF) in host defence against tuberculosis: implications for immunotherapies targeting TNF. Ann Rheum Dis. 2003;62(Suppl 2):37ii-42.

37. Harris J, Keane J. How tumour necrosis factor blockers interfere with tuberculosis immunity. Clin Exp Immunol. 2010;161(1):9.

38. Wergeland I, Pullar N, Assmus J, et al. IP-10 differentiates between active and latent tuberculosis irrespective of HIV status and declines during therapy. $J$ Infect. 2015;70(4):381-391.

39. Wergeland I, Assmus J, Dyrhol-Riise AM. Cytokine patterns in tuberculosis infection; IL-1ra, IL-2 and IP-10 differentiate borderline QuantiFERON-TB samples from uninfected controls. PLoS One. 2016;11(9):e0163848

40. Henke C, Marineili W, Jessurun J, et al. Macrophage production of basic fibroblast growth factor in the fibroproliferative disorder of alveolar fibrosis after lung injury. Am J Pathol. 1993;143(4): 1189-1199.

41. Awoniyi DO, Teuchert A, Sutherland JS, et al. Evaluation of cytokine responses against novel Mtb antigens as diagnostic markers for TB disease. J Infect. 2016;73(3):219-230.

42. Anbarasu D, Raja CP, Raja A. Multiplex analysis of cytokines/ chemokines as biomarkers that differentiate healthy contacts from tuberculosis patients in high endemic settings. Cytokine. 2013;61(3): 747-754.

43. Riou C, Perez Peixoto B, Roberts L, et al. Effect of standard tuberculosis treatment on plasma cytokine levels in patients with active pulmonary tuberculosis. PLoS One. 2012;7(5):e36886.
Journal of Inflammation Research

\section{Publish your work in this journal}

The Journal of Inflammation Research is an international, peer-reviewed open access journal that welcomes laboratory and clinical findings on the molecular basis, cell biology and pharmacology of inflammation including original research, reviews, symposium reports, hypothesis formation and commentaries on: acute/chronic inflammation; mediators of

\section{Dovepress}

inflammation; cellular processes; molecular mechanisms; pharmacology and novel anti-inflammatory drugs; clinical conditions involving inflammation. The manuscript management system is completely online and includes a very quick and fair peer-review system. Visit http://www.dove press.com/testimonials.php to read real quotes from published authors. 